University of Nebraska - Lincoln

DigitalCommons@University of Nebraska - Lincoln

\title{
The Relationship between the Perception of Axes of Symmetry and Spatial Memory during Early Childhood
}

Margaret R. Ortmann

University of Nebraska-Lincoln

Anne R. Schutte

University of Nebraska-Lincoln, aschutte2@unl.edu

Follow this and additional works at: https://digitalcommons.unl.edu/psychfacpub

Part of the Psychiatry and Psychology Commons

Ortmann, Margaret R. and Schutte, Anne R., "The Relationship between the Perception of Axes of Symmetry and Spatial Memory during Early Childhood" (2010). Faculty Publications, Department of Psychology. 495.

https://digitalcommons.unl.edu/psychfacpub/495

This Article is brought to you for free and open access by the Psychology, Department of at DigitalCommons@University of Nebraska - Lincoln. It has been accepted for inclusion in Faculty Publications, Department of Psychology by an authorized administrator of DigitalCommons@University of Nebraska - Lincoln. 
Published in Journal of Experimental Child Psychology 107:3 (November 2010), pp. 368-376; doi: 10.1016/j.jecp.2010.05.004 Copyright @ 2010 Elsevier Inc. Used by permission.

Submitted August 13, 2009; revised May 14, 2010; published online June 23, 2010.

\title{
The Relationship between the Perception of Axes of Symmetry and Spatial Memory during Early Childhood
}

\author{
Margaret R. Ortmann and Anne R. Schutte \\ Department of Psychology, University of Nebraska-Lincoln, Lincoln, NE 68588, USA \\ Corresponding author - A. R. Schutte, fax 402 472-4637, email aschutte2@unl.edu
}

\begin{abstract}
Early in development, there is a transition in spatial working memory (SWM). When remembering a location in a homogeneous space (e.g., in a sandbox), young children are biased toward the midline symmetry axis of the space. Over development, a transition occurs that leads to older children being biased away from midline. The dynamic field theory (DFT) explains this transition in biases as being caused by a change in the precision of neural interaction in SWM and improvements in the perception of midline. According to the DFT, young children perceive midline, but there is a quantitative improvement in the perception of midline over development. In the experiment reported here, children and adults needed to determine on which half of a large monitor a target was located. In support of the DFT, even the youngest children performed above chance at most locations, but performance also improved gradually with age.
\end{abstract}

Keywords: spatial cognition, spatial memory, symmetry, perception, dynamic systems, development

\section{Introduction}

Spatial memory develops early. Even infants are capable of remembering locations (e.g., Newcombe, Huttenlocher, \& Learmonth, 1999), but spatial memory continues to develop into childhood (e.g., [Huttenlocher et al., 1994; Spencer and Hund, 2003). One aspect of spatial memory that develops during childhood is children's use of symmetry axes as reference axes (Huttenlocher et al., 1994; Schutte and Spencer, 2002; Schutte and Spencer, in press), but what leads to this development? One possibility is that there are changes in children's perception of symmetry axes. The current study examines the ability of children to 
perceive the symmetry axis of a space.

Several studies have found a developmental transition in spatial working memory (SWM) biases in tasks that used a homogeneous space (Huttenlocher et al., 1991; Huttenlocher et al., 1994; Schutte and Spencer, 2002; Schutte and Spencer, in press). For example, in the spaceship SWM task (Schutte \& Spencer, 2002), children were presented with a spaceship-shaped target on a homogeneous tabletop, and after a delay they pointed to where they remembered the target to be. In this task, the vertical symmetry axis, or midline, of the task space acted as a reference axis. At around 3 years of age, children's memory responses were biased toward midline for a wide range of hiding locations (Huttenlocher et al., 1994; Schutte et al., 2010; Schutte and Spencer, 2002). Later in development, children's responses were biased away from midline (Hund and Spencer, 2003; Huttenlocher et al., 1994; Schutte et al., 2010; Spencer and Hund, 2003). This transition in geometric biases occurred gradually, and when the transition occurred depended on target location (Schutte \& Spencer, in press).

The dynamic field theory (DFT), a dynamic systems model of SWM made up of a network of dynamic neural fields, has been used to explain this transition (Schutte and Spencer, in press; Simmering et al., 2008). Simulations of the model using the older 3-year-old parameter set and the 6-year-old parameter set from Schutte and Spencer (in press) are shown in Figure 1A and B, respectively. The model is made up of three interconnected fields: an excitatory perceptual field (PF, first layer) that codes the perceptual structure in the task space, an excitatory SWM field (SWM, third layer) that receives excitatory input from the perceptual field (see solid arrows) and maintains the memory of the target location, and a field of inhibitory interneurons (Inhib, second layer) that receives input from both the perceptual and SWM fields (see solid arrows) and sends inhibition back to both fields (see dashed arrows). For each field, location is represented along the $x$ axis, activation along the $y$ axis, and time along the $z$ axis. Neurons in each field send positive activation to nearby neurons and, through the inhibitory field, send inhibition to neurons farther away. The result of these interactions is a form of local excitation/lateral inhibition that allows the SWM field to sustain a peak of activation in the absence of input.

Input into the perceptual field, which marks the location of the reference axis, influences the target peak in the SWM field. When the target peak overlaps with excitatory input from the midline reference axis, the target peak drifts toward the input (Figure 1A). If, however, the target peak overlaps with the inhibition from the reference axis, the peak drifts away from it (Figure 1B). According to the spatial precision hypothesis (Schutte and Spencer, in press; Schutte et al., 2003; Simmering et al., 2008), over development the model changes in two ways. First, neural interaction becomes stronger and, as a result, peaks become narrower and more stable. Second, the perception of midline becomes more precise; that is, the reference input to the perceptual field becomes narrower and stronger. The transition in geometric biases occurs gradually as the result of these two quantitative changes (Schutte and Spencer, in press; Schutte and Spencer, 2009).

In addition to explaining performance in SWM tasks, the DFT has been applied to position discrimination tasks (Simmering and Spencer, 2008; Simmering et al., 2006). Simmering and colleagues have shown that the DFT and the spatial precision hypothesis can account for children's and adults' performance in position discrimination tasks. In these tasks, participants are shown two targets in succession and determine whether the targets are in the same or different locations. Based on the DFT, Simmering and colleagues (2006) predicted that adults would perform more accurately when the targets were closer to midline and when the second target appeared toward midline relative to the first target. In contrast, Simmering and Spencer (2008) predicted that young children would be more accurate when the second target was away from midline. Results supported these predictions and demonstrated the generalizability of the DFT to discrimination tasks.

The research presented here tested predictions of the DFT for another type of position discrimination task, namely the discrimination of a target location relative to midline. According to the DFT, over development the precision with which children perceive midline improves (Schutte and Spencer, 2002; Schutte and Spencer, in press; Simmering et al., 2008). The graphs shown to the right of Figure 1A and B show a section of the perceptual field. In the 3-year-old model, the midline reference axis activation in the PF is weak and broad, whereas the midline activation for the 6-year-old model is stronger and narrower. Thus, according to the DFT, 6-year-olds should do better than 3-year-olds at discriminating locations relative to midline. This improvement should be gradual between 3 and 6 years of age because in the DFT the perception of midline changes gradually (Schutte and Spencer, in press; Schutte and Spencer, 2009). 

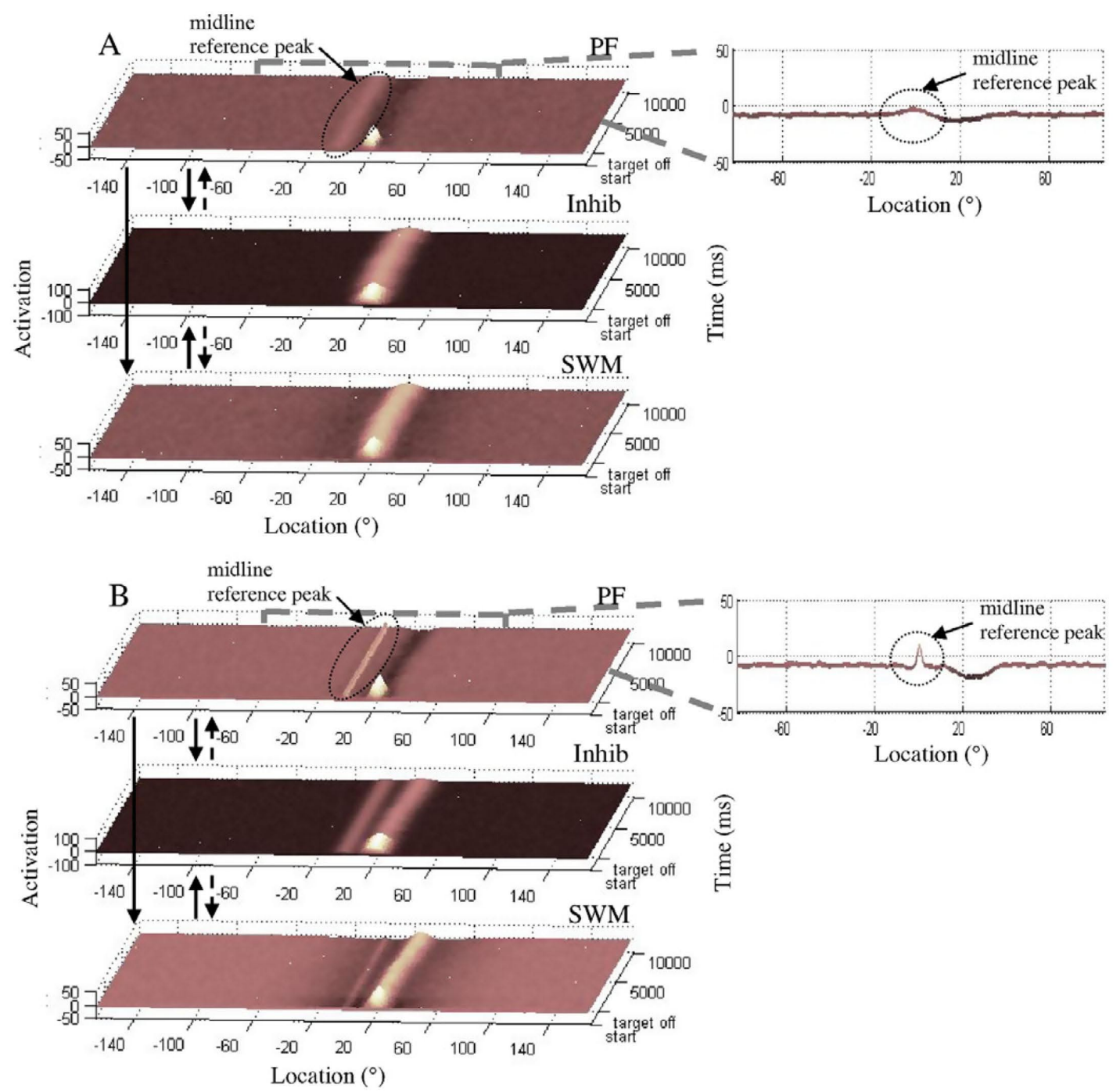

Figure 1. Simulations of the DFT: (A) 3-year-old model; (B) 6-year-old model. PF, perceptual field; Inhib, inhibitory field; SWM, excitatory spatial working memory field. Arrows represent interaction between fields. Solid arrows represent excitatory connections, and dashed arrows represent inhibitory connections. In each field, location is represented along the $x$ axis (with midline at location 0 ), activation along the $y$ axis, and time along the $z$ axis. The trial begins at the front of the figure and moves toward the back. Time slices from the end of the delay for the perceptual field are shown on the right. See text for additional details.

Previous research has found that the perception of symmetry develops at a young age (e.g., Bornstein, Ferdinandsen, \& Gross, 1981). Developmental studies of symmetry have examined the detection of symmetrical versus asymmetrical objects or patterns (e.g., [Bornstein and Stiles-Davis, 1984; Bornstein et al., 1981) or have focused on the development of a preference for symmetrical objects (Royer, 1981). Studies have found that the perception of symmetry develops during infancy (e.g., Bornstein \& Krinsky, 1985), with the perception of vertical symmetry developing first (Bornstein et al., 1981). Previous research, however, has not examined how accurately children can localize a symmetry axis. It is possible that for young children the location of the axis may be "fuzzy," especially in cases where there are no symmetry cues near the reference axis, as is the case in large task spaces such as a large monitor. The current study investigated developmental changes in the perception of midline by asking children to view a target on a large monitor and determine on which half of the monitor the target was located. 


\section{Method}

\section{Participants}

Participants were 15 3-year-olds (9 boys and 6 girls, mean age $=3.6$ years, $S D=4.6$ months), 154 year-olds ( 7 boys and 8 girls, mean age $=4.6$ years, $S D=4.1$ months), 18 -year-olds (5 boys and 13 girls, mean age $=5.6$ years, $S D=3.1$ months), 15 -year-olds (6 boys and 9 girls, mean age $=6.6$ years, $S D=3.7$ months), and 15 adults ( 4 men and 11 women, mean age $=22$ years, $S D=1$ year 2 months). An additional 6 children came into the lab but were not included in the analyses due to unwillingness to finish the game (2 3-year-olds) or to not answering at least three of the four control trials correctly (3 3-yearolds and 14 -year-old) (see below for details). Parents and adult participants gave informed consent. Children were given $\$ 10$ and a small toy as compensation, and adult participants received course credit.

\section{Apparatus}

Participants viewed the stimuli on a $29 \times 42$-inch $(74 \times 107-\mathrm{cm})$ LCD monitor with a screen resolution of $1024 \times 768$ pixels. The monitor was tilted $15^{\circ}$ from horizontal. The software program E-Prime was used to run the task. The stimuli were small smiley faces $(1.2 \mathrm{~cm}$ wide $\times 0.8 \mathrm{~cm}$ tall).

\section{Procedure}

Children started the session with training trials. They were told that two identical twins lived in their own yards and did not cross into each other's yard. A piece of paper represented the "yards," with each twin "living" on half of the paper. A transparency with a line drawn down the center was laid over the paper to show children how the two twins had their own yards. After explaining the rules of the task, the experimenter placed a smiley face on the sheet of paper and asked each participant to identify which twin it was by moving a joystick to the left or right to indicate the yard in which the smiley face was located. The transparency was then laid down to provide feedback about the location. The child completed a minimum of three training trials. The order was random, but each side of the paper was used at least once. If the child was not correct on at least two of the three training trials, trials were added until the child answered two in a row correctly. In general, children did not require more than three training trials.

After training, the experimenter completed a demonstration trial on the large monitor, followed by two practice trials. The smiley face was presented, and the participant was asked to respond. The smiley face stayed on the monitor until the participant responded. This was done to avoid introducing a spatial memory component. After each trial, a yellow line on midline appeared along with the target, and the participant was told whether or not he or she was correct. If the participant was incorrect, he or she was told "nice try" and encouraged to try again.

Adults started the procedure at the monitor. They were told that they needed to move the joystick to either the right or left depending on where they saw the smiley face. The same demonstration trial and two practice trials were conducted.

There were two trials to each target location. The targets were $\pm 0.654, \pm 1.307, \pm 2.605, \pm 3.882, \pm 5.130$, and $\pm 11.491 \mathrm{~cm}$ from midline, with positive locations to the right and negative locations to the left. There were a total of 24 randomly ordered trials. The $\pm 11.491-\mathrm{cm}$ location was a control location. Data from participants who did not respond correctly on three of four control trials were not included in the analyses.

\section{Results}

The mean number of correct responses to each distance from midline, for each age group, can be seen in Figure 2. The distance from midline is on the $x$ axis, and the number of correct responses is on the $y$ axis. The maximum number correct is 4.0, and chance performance is 2.0. Performance of all ages was above chance for the locations farthest from midline, and performance decreased as the targets approached midline. 


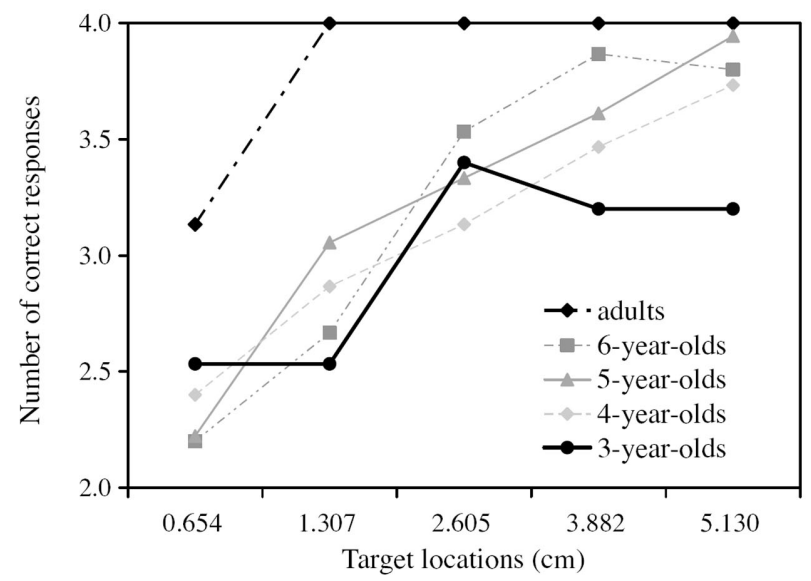

Figure 2. Results for each distance from midline. Note that 2.0 is chance performance and 4.0 is perfect performance.

We conducted an analysis of variance (ANOVA) with side of the monitor (left or right) and location $(0.654,1.307,2.605,3.882$, or $5.130 \mathrm{~cm})$ as within-participants factors and with age (3 years, 4 years, 5 years, 6 years, or adult) as a between-participants factor. There was a significant side main effect, $F(1,73)=6.173$, $p<.025$. Overall performance was better on the right than on the left (right: $M=1.57$; left: $M=1.70$ ). There was also a significant location main effect, $F(4,292)=40.225, p<.001$. Correct performance decreased as location approached midline (see Figure 2). There was also a significant Location $\times$ Age interaction, $F(16,292)=1.967, p<.025$, and a significant age main effect, $F(4,73)=9.253, p<.001$. Correct performance increased as age increased (see Figure 3). Follow-ups of the Age $\times$ Location interaction examined the age effect at each target location. At the location closest to midline, there was only a marginal age effect, $F(4,77)=2.304, p=.07$. There was a significant age effect at the other locations $-5.130 \mathrm{~cm}: F(4,77)=4.985$, $p=.001 ; 3.882 \mathrm{~cm}: F(4,77)=3.938, p<.01 ; 2.605 \mathrm{~cm}: F(4,77)=3.142, p<.025 ; 1.307 \mathrm{~cm}: F(4,77)=7.126$, $p<.001$. Thus, performance across ages differed significantly at all locations except the one closest to midline.

To determine which ages differed from each other, we performed follow-up $t$ tests (two-tailed) comparing ages at the locations with a significant age effect. At $5.130 \mathrm{~cm}$, the 3-year-olds differed significantly from the 4 - and 5 -year-olds -3 and 4 years: $t(28)=-2.117, p<.05 ; 3$ and 5 years: $t(31)=-3.520$, $p=.001-$ and differed marginally from the 6-year-olds, $t(28)=-2.005, p=.055$. The performance of the 4 , 5-, and 6-year-olds did not differ at this location (all ps > .09). The 3- and 4-year-olds' performance differed significantly from that of the adults -3 years and adults: $t(28)=-3.595, p=.001 ; 4$ years and adults: $t(28)=-2.256, p<.05$. The 5 - and 6-year-olds did not differ significantly from the adults (all $p$ s $>.10$ ).

At $3.882 \mathrm{~cm}$, the 3-year-olds differed significantly from the 6-year-olds, $t(28)=-3.035, p=.005$. All other comparisons among the 3-, 4-, 5-, and 6-year-olds were not significant (all ps > .09). The 3-, 4-, and 5-yearolds differed significantly from the adults -3 years and adults: $t(28)=-4.583, p<.001 ; 4$ years and adults: $t(28)=-2.477, p<.025 ; 5$ years and adults: $t(31)=-2.153, p<.05$. In contrast, the 6-year-olds did not differ from the adults, $t(28)=-1.000, n s$.

The performance of the 3-, 4-, 5-, and 6-year-olds did not differ significantly at $2.605 \mathrm{~cm}$ (all ps >.10). The 3-, 4-, and 5-year-olds all differed significantly from the adults -3 years and adults: $t(28)=-3.154$, $p<.005$; 4 years and adults: $t(28)=-4.026, p<.001$; 5 years and adults: $t(31)=-3.754, p=.001-$ whereas the 6-year-olds did not differ from the adults, $t(28)=-1.974, n s$.

At $1.307 \mathrm{~cm}$, the performance of the 3-, 4-, 5-, and 6-year-olds did not differ significantly from each other (all ps $>.10$ ), whereas the performance of all ages differed significantly from that of the adults 3 years and adults: $t(28)=-6.205, p<.001 ; 4$ years and adults: $t(28)=-4.795, p<.001 ; 5$ years and adults: $t(31)=-3.891, p<.001 ; 6$ years and adults: $t(28)=-5.292, p<.001$. 


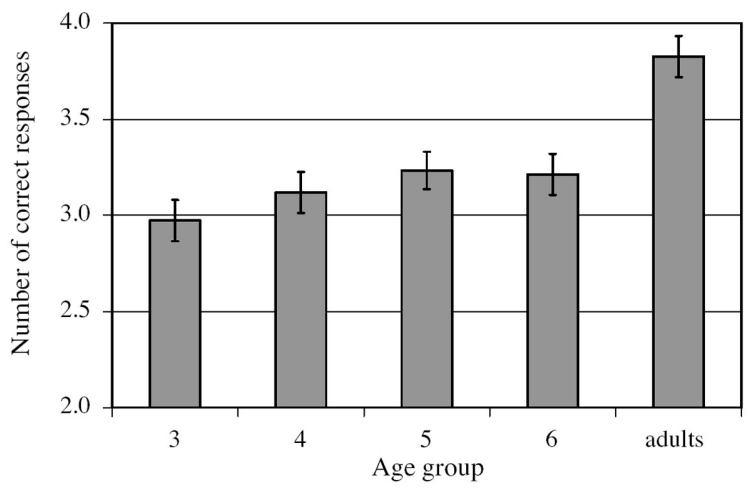

Figure 3. Average number of correct responses for each age. Note that 2.0 is chance performance. Error bars represent the standard error of the mean.

Table 1. $t$ Tests for each age group comparing performance at each location with chance.

\begin{tabular}{|c|c|c|}
\hline Age and location $(\mathrm{cm})$ & $d f$ & $t$ \\
\hline \multicolumn{3}{|l|}{ 3-year-olds } \\
\hline 0.654 & 14 & $2.26^{*}$ \\
\hline 1.307 & 14 & $2.26^{*}$ \\
\hline 2.605 & 14 & $7.36^{* *}$ \\
\hline 3.882 & 14 & $6.87^{* *}$ \\
\hline 5.130 & 14 & $5.39 * *$ \\
\hline \multicolumn{3}{|l|}{ 4-year-olds } \\
\hline 0.654 & 14 & 1.57 \\
\hline 1.307 & 14 & $3.67^{*}$ \\
\hline 2.605 & 14 & $5.26^{* *}$ \\
\hline 3.882 & 14 & $6.81^{* *}$ \\
\hline 5.130 & 14 & $14.67^{* *}$ \\
\hline \multicolumn{3}{|l|}{ 5-year-olds } \\
\hline 0.654 & 17 & 0.81 \\
\hline 1.307 & 17 & $4.78^{* *}$ \\
\hline 2.605 & 17 & $8.25^{* *}$ \\
\hline 3.882 & 17 & $9.80^{* *}$ \\
\hline 5.130 & 17 & $35.00^{* *}$ \\
\hline \multicolumn{3}{|l|}{ 6-year-olds } \\
\hline 0.654 & 14 & 1.00 \\
\hline 1.307 & 14 & $2.65^{*}$ \\
\hline 2.605 & 14 & $6.49^{* *}$ \\
\hline 3.882 & 14 & $14.00^{* *}$ \\
\hline 5.130 & 14 & $9.00^{* *}$ \\
\hline \multicolumn{3}{|l|}{ Adults } \\
\hline 0.654 & 14 & $4.43^{* *}$ \\
\hline 1.307 & & - \\
\hline 2.605 & & - \\
\hline 3.882 & & - \\
\hline 5.130 & & - \\
\hline
\end{tabular}

A dash (-) indicates that $t$ was not computed due to perfect performance.

${ }^{*} p<.05 ;{ }^{* *} p<.01$. 
To determine whether performance was better than chance, we conducted planned $t$ tests. Results of the $t$ tests are presented in Table 1 . Note that the adults' performance at the four locations farthest from midline was perfect, so $t$ values could not be computed. Performance at all locations was above chance for the 3year-olds and adults. For 4-, 5-, and 6-year-olds, all locations except the closest location were above chance.

In summary, performance of the children declined as locations approached midline. Adults, on the other hand, were perfect at all locations except the closest one. Performance of the 3-year-olds differed significantly from that of the other age groups, but there were no differences among the 4-, 5-, and 6-yearolds. All ages differed significantly from the adults at one or more locations.

\section{Discussion}

Between 3 and 6 years of age, there were small developmental changes in the ability to categorize locations around midline. Specifically, there was a small but significant increase in the number of targets categorized correctly. According to the DFT, the transition in the direction of geometric biases occurs due to two developmental changes: stronger neural interactions and changes in the perception of the symmetry axis. The results of this study support the DFT's proposal that even young children are able to perceive the symmetry axis and over development there is a gradual quantitative change in the precision of their perception (Schutte \& Spencer, in press). Interestingly, the largest change was not between 3 and 4 years of age, which is when the transition in geometric biases occurs. Instead, the largest change in performance occurred between childhood and adulthood, which was also the largest age difference in the study. This result fits with the DFT's proposal that the changes in the perception of midline occur gradually (Schutte and Spencer, in press; Schutte and Spencer, 2009).

Another theory of spatial memory prevalent in the literature is the category adjustment model (CAM) (Huttenlocher et al., 1991; Huttenlocher et al., 1994). Although the CAM was not proposed to explain categorization in perceptual tasks, it has been used to explain the transition in geometric biases, so it is useful to examine whether these data have implications for the model. The CAM is a Bayesian model that proposes that geometric biases arise out of processes that increase the average accuracy of the estimates of locations (Huttenlocher, Hedges, Corrigan, \& Crawford, 2004). According to the CAM (Huttenlocher et al., 1991; Huttenlocher et al., 1994), as it has been applied to spatial memory, children and adults encode two types of information: categorical and fine-grained. Categorical information relates to the spatial category in which the object is located. Fine-grained information is an inexact unbiased memory of the location. When a location is presented, people encode both types of information. At recall, people combine the fine-grained and categorical information (i.e., the prototype at the center of the category) and are biased toward the prototype. In the CAM, the transition in geometric biases is the result of a change in categorization (Huttenlocher et al., 1994). Young children categorize the space as one category, whereas older children and adults divide the space into two categories.

According to Huttenlocher, Hedges, Crawford, and Corrigan (2007), boundaries influence bias through truncation. As boundaries become uncertain, the effect of truncation reduces due to more variable boundary locations and, as a result, bias away from boundaries decreases (Huttenlocher et al., 1991). In addition, as boundaries become uncertain, variability of responses and absolute error increase. How does this apply to changes over development? It is not completely clear in the model how children transition from one category to two categories (see Schutte \& Spencer, in press, for a discussion), but it is possible that initially perception of the midline is so uncertain that treating the space as one category leads to lower error than treating it as two categories. The findings of a study by Huttenlocher and colleagues (2004) using a circledot task support the proposal that people do not use uncertain boundaries. In the experiment, adults were taught to categorize locations based on diagonal boundaries. Despite the training, when they reproduced the locations from memory, their biases reflected vertical and horizontal boundaries.

Wedell and colleagues (Fitting et al., 2005; Fitting et al., 2007; Wedell et al., 2007) proposed a revision to the CAM called the fuzzy boundary model. According to this model, bias near boundaries reduces due to the prototype from the incorrect category being recruited on some trials (see also Huttenlocher et al., 2004). The results of the current study suggest that this could happen at some locations. Even adults were not perfect at determining the category of the closest location, suggesting that it could be miscategorized on some trials. The adults, however, were better than children at categorizing many locations. Therefore, 
according to the fuzzy boundary model, children's responses should be less biased than those of adults due to children's miscategorization. Schutte and Spencer (in press) found that children at the transition point in geometric biases were not significantly biased, and this could be caused by miscategorization. Children, however, were biased away from midline when the targets were $20^{\circ}$ from midline before they were biased away when the targets were $30^{\circ}$ or $50^{\circ}$ from midline. This result is not what would be predicted if the lack of bias were caused by miscategorization. The fuzzy boundary model has not been applied to development, however, so it is unclear how it would account for the pattern of bias seen during the transition.

One limitation of the current study is that the structure of the task involved asking children to categorize the location of the target rather than determining how they would spontaneously categorize the location. It is possible that when 3-year-olds are confronted with an SWM task, they do not spontaneously subdivide the space into two categories even when they are capable of subdivision. There are several differences between SWM tasks and the discrimination task used here that could potentially lead to differences in subdivision. Future work will examine whether children's subdivision ability is predictive of their geometric biases in SWM tasks.

In conclusion, this study is a significant first step in examining children's ability to subdivide space. The gradual improvement in the perception of midline supports the DFT's explanation that the transition in geometric biases is partially due to quantitative improvements in the ability to perceive symmetry axes (Schutte \& Spencer, in press). Now that a method for examining young children's ability to localize symmetry axes has been developed, future research can examine in more detail the connection between the perception of midline and SWM.

\section{Acknowledgments}

This research was funded by NICHD R03 HD053359 awarded to Anne R. Schutte. The authors thank the children and parents who participated in this research. The authors also thank John P. Spencer and three anonymous reviewers for their comments on an earlier version of this article.

\section{References}

Bornstein et al., $19814 \mathrm{M}$. H. Bornstein, K. Ferdinandsen, and C. G. Gross, Perception of symmetry in infancy, Developmental Psychology 17 (1981), pp. 82-86.

Bornstein and Krinsky, 19854 M. H. Bornstein and S. J. Krinsky, Perception of symmetry in infancy: The salience of vertical symmetry and the perception of pattern wholes, Journal of Experimental Child Psychology 39 (1985), pp. 1-19.

Bornstein and Stiles-Davis, $1984 \varangle$ M. H. Bornstein and J. Stiles-Davis, Discrimination and memory for symmetry in young children, Developmental Psychology 20 (1984), pp. 637-649.

Fitting et al., 20054 S. Fitting, D. H. Wedell, and G. L. Allen, Memory for spatial location: Influences of environmental cues and task field rotation. In: A. G. Cohn and D. M. Mark, Editors, COSIT 2005, LNCS 3693, Springer, Berlin, Germany (2005), pp. 459-474.

Fitting et al., $2007<$ S. Fitting, D. H. Wedell, and G. L. Allen, Memory for spatial location: Cue effects as a function of field rotation, Memory \& Cognition 35 (2007), pp. 1641-1658.

Hund and Spencer, 20034 A. M. Hund and J. P. Spencer, Developmental changes in the relative weighting of geometric and experience-dependent location cues, Journal of Cognition and Development 4 (2003), pp. 3-38.

Huttenlocher et al., $2004<$ J. Huttenlocher, L. V. Hedges, B. Corrigan, and L. E. Crawford, Spatial categories and the estimation of location, Cognition 93 (2004), pp. 75-97.

Huttenlocher et al., $2007<$ J. Huttenlocher, L. V. Hedges, L. E. Crawford, and B. Corrigan, Estimating stimuli from contrasting categories: Truncation due to boundaries, Journal of Experimental Psychology: General 136 (2007), pp. 502-519.

Huttenlocher et al., $1991<$ J. Huttenlocher, L. V. Hedges, and S. Duncan, Categories and particulars: Prototype effects in estimating spatial location, Psychological Review 98 (1991), pp. 352-376.

Huttenlocher et al., $1994<\mathrm{J}$. Huttenlocher, N. Newcombe, and E. H. Sandberg, The coding of spatial location in young children, Cognitive Psychology 27 (1994), pp. 115-147. 
Newcombe et al., $1999 \varangle$ N. Newcombe, J. Huttenlocher, and A. Learmonth, Infants' coding of location in continuous space, Infant Behavior \& Development 22 (1999), pp. 483-510.

Royer, 19814 F. L. Royer, Detection of symmetry, Journal of Experimental Psychology: Human Perception and Performance 7 (1981), pp. 1186-1210.

Schutte et al., 2010, 4 A. R. Schutte, B. K. Keiser, M. R. Ortmann, and C. Kobza-Guerrero, The impact of attentional shifts on spatial memory in early childhood. Unpublished manuscript, University of Nebraska-Lincoln, 2010.

Schutte and Spencer, 20024 A. R. Schutte and J. P. Spencer, Generalizing the dynamic field theory of the A-not-B error beyond infancy: Three-year-olds' delay- and experience-dependent location memory biases, Child Development 73 (2002), pp. 377-404.

Schutte and Spencer, 20094 A. R. Schutte and J. P. Spencer, Tests of the dynamic field theory and the spatial precision hypothesis: Capturing a qualitative developmental transition in spatial working memory, Journal of Experimental Psychology: Human Perception and Performance 35 (2009), pp. 1698-1725.

Schutte and Spencer, in press, 4 A. R. Schutte and J. P. Spencer, The influence of perceptual structure on a developmental transition in spatial working memory. Journal of Cognition and Development; in press.

Schutte et al., $2003 \varangle$ A. R. Schutte, J. P. Spencer, and G. Schöner, Testing the dynamic field theory: Working memory for locations becomes more spatially precise over development, Child Development 74 (2003), pp. 1393-1417.

Simmering et al., $2008<$ V. R. Simmering, A. R. Schutte, and J. P. Spencer, Generalizing the dynamic field theory of spatial cognition across real and developmental time scales, Brain Research 1202 (2008), pp. 68-86.

Simmering and Spencer, $2008<$ V. R. Simmering and J. P. Spencer, Generality with specificity: The dynamic field theory generalizes across tasks and time scales, Developmental Science 11 (2008), pp. 541-555.

Simmering et al., 20064 V. R. Simmering, J. P. Spencer, and G. Schöner, Reference-related inhibition produces enhanced position discrimination and fast repulsion near axes of symmetry, Perception \& Psychophysics 68 (2006), pp. 1027-1046.

Spencer and Hund, $2003<$ J. P. Spencer and A. M. Hund, Developmental continuity in the processes that underlie spatial recall, Cognitive Psychology 47 (2003), pp. 432-480.

Wedell et al., $2007 \varangle$ D. H. Wedell, S. Fitting, and G. L. Allen, Shape effects on memory for location, Psychonomic Bulletin \& Review 14 (2007), pp. 681-686. 